\title{
Global analysis of all linear stable settings of a storage ring lattice
}

\author{
David S Robin, Weishi Wan, and Fernando Sannibale \\ Lawrence Berkeley National Laboratory, 1 Cyclotron Road Mail Stop 80R0114, Berkeley, California 94720-8229, USA
}

Victor P. Suller

CAMD/LSU, 6980 Jefferson Highway, Baton Rouge, Louisiana 70806, USA

(Received 29 October 2007; published 28 February 2008)

\begin{abstract}
The traditional process of designing and tuning the magnetic lattice of a particle storage ring lattice to produce certain desired properties is not straightforward. Often solutions are found through trial and error and it is not clear that the solutions are close to optimal. This can be a very unsatisfying process. In this paper we take a step back and look at the general stability limits of the lattice. We employ a technique we call GLASS (GLobal scan of All Stable Settings) that allows us to rapidly scan and find all possible stable modes and then characterize their associated properties. In this paper we illustrate how the GLASS technique gives a global and comprehensive vision of the capabilities of the lattice. In a sense, GLASS functions as a lattice observatory clearly displaying all possibilities. The power of the GLASS technique is that it is fast and comprehensive. There is no fitting involved. It gives the lattice designer clear guidance as to where to look for interesting operational points. We demonstrate the technique by applying it to two existing storage ring lattices - the triple bend achromat of the Advanced Light Source and the double bend achromat of CAMD. We show that, using GLASS, we have uncovered many interesting and in some cases previously unknown stability regions.
\end{abstract}

DOI: 10.1103/PhysRevSTAB.11.024002

PACS numbers: 41.85.-p, 29.20.db

\section{INTRODUCTION}

Assume that one has a storage ring and wants to adjust the lattice settings to obtain certain properties or a combination of properties such as low emittance, small momentum compaction, high brightness, small beam size, etc. Finding settings that will provide the desired properties is not straightforward and can be a very difficult. It is in fact much more difficult than the inverse problem where the optical elements are given and one is asked to compute the resulting properties of the beam. Going from the properties and finding the settings is a nonlinear problem with many local minima.

In many ways finding a solution is an art which is aided by the instincts and experience of the practitioner. Traditionally, the approach is to first find a stable solution (often by trial and error) that roughly meets the desired properties and then to locally optimize around it. This traditional approach has several weaknesses. In particular, the process can be slow and does not guarantee that one has obtained an optimal solution.

What is desirable is to be able to obtain a global understanding or view of the lattice to rapidly guide one towards optimal solutions. For very simple lattices such as a focus/ defocus cell, it is possible to analytically determine the entire linear stability region-the so-called necktie diagram [1]. For somewhat more complicated lattices, scans of quadrupole settings have been done to help get a wideranging feel for the different stability regions [2,3]. With the increase in computer speed, global scans have become more practical. In this paper we extend this idea of performing wide scans and present a technique that we have developed that rapidly identifies stable regions, computes lattice properties, and provides a global view of the lattice. This technique takes the guesswork out of the optimization process, making it more transparent and efficient.

Having a method of gaining a global understanding of a lattice is useful and can significantly guide the lattice designer. The technique that we use to search for different operational modes is straightforward and powerful. Yet, to our knowledge, this approach has not been fully exploited before.

\section{GLASS TECHNIQUE}

In this approach, instead of trying to fit the lattice to find specific properties, we follow the steps: (i) Scan ALL possible quadrupole settings; (ii) find ALL stable settings; (iii) compute properties of ALL stable settings; (iv) filter by property those settings that may be of interest.

At the end of the process one has a database with all possible solutions and associated properties. Then, by querying the database against certain properties, it is possible to find any and all lattice settings that satisfy the properties. In addition, the data can be viewed such as to give a global understanding of the lattice. We call this technique GLobal scan of All Stable Settings or GLASS.

At first, it seems impractical to scan all possible lattice settings. In fact for relatively simple lattices, it is not only possible but also practical. We illustrate this with two examples: (1) the Advanced Light Source (ALS) which has a triple bend (TBA) achromat structure and (2) the CAMD Light Source which has a double bend (DBA) structure. Both of the examples that were chosen are 
representative of TBA and DBA lattice structures that are amongst the most common lattice structures used in storage ring lattices. This analysis could just as easily have been performed on other lattice structures such as a theoretical minimum emittance lattice and other more complex lattice structures. This will be discussed later in the paper.

\section{A. Example 1-Triple bend achromat of the ALS}

The entire ALS storage ring consists of 12 sectors. Each sector is a simple triple bend achromat (TBA) structure. The TBA structure is used in other storage rings such as the Swiss Light Source, the Pohang Light Source, the Taiwan Light Source, and others. The ALS lattice, in particular, has been well studied over many years. Figure 1 shows the layout of the ALS sector and the lattice functions in the nominal operational mode. The basic ALS sector is a mirror symmetric structure consisting of 3 families of quadruples-QF, QD, and QFA. The present setting of the ALS sector nominally operates at tunes $\left(\nu_{x}, \nu_{y}\right)=$ $(1.19,0.77)$ and $\eta=0.06[\mathrm{~m}]$ (dispersion at the center of the long straight sections) and at a nominal energy of $1.9 \mathrm{GeV}$.

Besides the nominal mode, the lattice has been operated for users in a few other modes-zero dispersion in the straights and at a vertical tune of 8.2 where the vertical $\beta$-function is larger ( $4 \mathrm{~m}$ compared with $2.5 \mathrm{~m}$ in the center of the straights). In special operation shifts, the lattice has also been operated in a large emittance zero momentum compaction mode. Recent theoretical studies have shown that it is possible to significantly reduce the emittance from the present operational mode [4].

Are there other interesting operational modes that can be supported by this lattice? For instance, could one operate

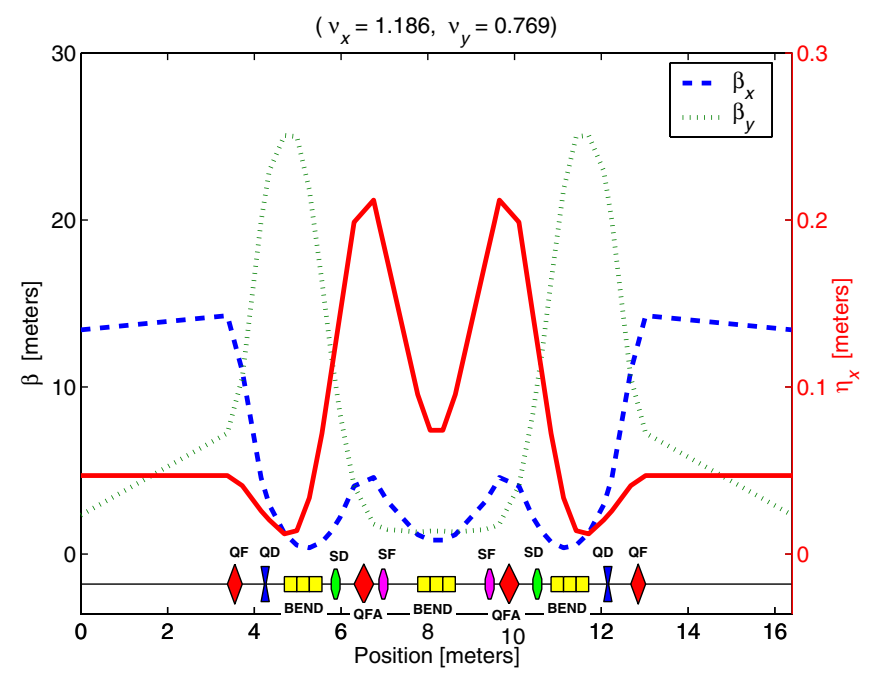

FIG. 1. (Color) Lattice of one of 12 ALS sectors and associated lattice functions $\left(\beta_{x}, \beta_{y}\right.$, and $\left.\eta_{x}\right)$ in the nominal operational mode starting from the center of one straight section and going to the center of the following straight section. with a lattice with a small emittance and a small $\beta$-function in the straights? Or are there modes that have both a small emittance and a small momentum compaction factor? We have applied GLASS to see how these questions can be answered.

\section{Finding the ALS stable regions}

The first step in the GLASS process is to scan the quadrupole families over a wide range. For all 3 quadrupole families, the magnet strengths $k_{\mathrm{QF}}, k_{\mathrm{QD}}$, and $k_{\mathrm{QFA}}$ are each scanned over 1000 settings ranging between $k=$ $-10 \mathrm{~m}^{-2}$ and $10 \mathrm{~m}^{-2}$. This large range covers the full operational capabilities of the ALS power supplies. All together, there are $1 \times 10^{9}$ combinations scanned. For each of these combinations, the 4D linear transfer matrix is computed and determined to be stable [5], by requiring the trace of the $4 \times 4$ transfer matrix in both the horizontal and vertical planes to be less than 2 . The computation was done using the code COSY [6] but can be done quickly using any other matrix multiplication code. The total time to scan all possible settings is less than one day on a 64 bit Linux box. This process is a little time consuming but only needs to be done once.

Of the billion initial combinations, approximately half a million were stable. Each stable setting is recorded and plotted in Fig. 2. As seen in Fig. 2, the stable points tend to group in different regions. There are many $(>13)$ distinct regions where stable solutions exist. We have enumerated several different stable regions in Fig. 2. The nominal ALS lattice setting resides in region 1.

Already, by performing this first step in GLASS of finding the linear stable solutions, we are seeing something interesting. First, there are many stable regions - many of which were not previously known. Before doing the scan, we had known about and operated the ring in only two regions-regions 1 and 10 in Fig. 2. Second, the space of stable solutions is sparse $(<1 / 1000)$ so without doing a

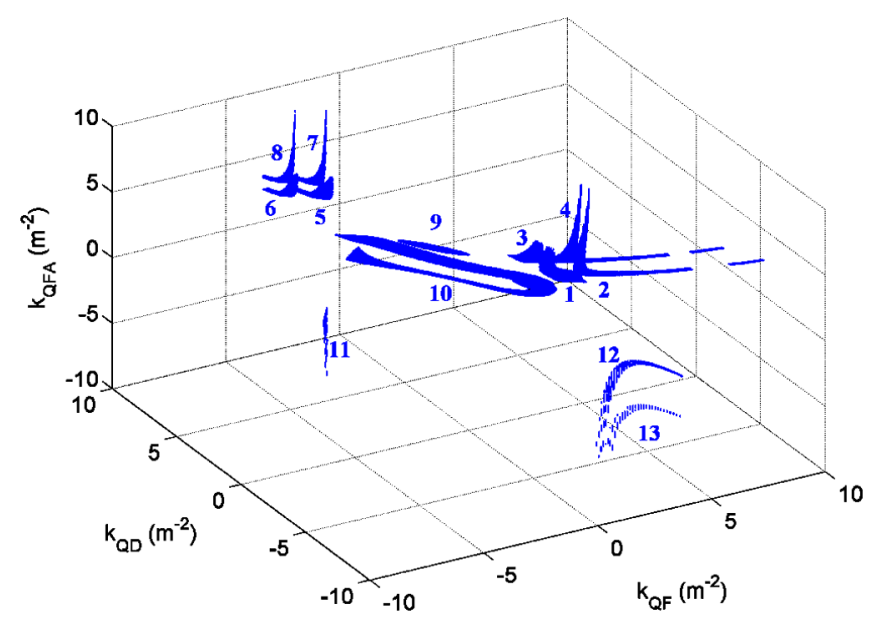

FIG. 2. (Color) All linear stable solutions in $k$-space for one of the 12 ALS TBA sectors. 
scan it would be difficult to locate them. Most of these regions are far from any that had been previously explored or even known about.

It is important to note that the constraint of linear stability is a necessary but not sufficient condition for operation of storage ring. Not all lattices found are feasible. Other considerations such as large amplitude and energy stability (i.e. dynamic aperture) need to be considered. The GLASS results contain all possible practical results as well as some impractical results.

\section{Computing the properties of all stable settings}

The next step in the GLASS process is to compute relevant properties for each of these stable settings. For instance, one can compute the tunes, momentum compaction, emittance, dispersion, beam sizes at the source points, etc. [7] — whatever may be of interest. This takes very little time (minutes). After this is completed we have a global database containing each of the linear stable settings and associated properties.

From this data, one can now query the database against different properties. For instance, one can search for all solutions where the emittance is small. Or one can look for all solutions where the momentum compaction is small. This requires no fitting or guess work. All possible solutions are found.

Querying the database for multiple properties is effective for rapidly locating optimal solutions to a broad set of questions. For instance, one could search for all lattices that have a low emittance and a small horizontal $\beta$-function in the straights, and a large vertical $\beta$-function in the arcs, etc. The result is fast and comprehensive. In fact, the ability to query the database solves the difficult inverse optical problem of finding the settings that will generate certain desired properties. Now we will illustrate the usefulness of the technique using two examples lattices with small emittance and lattices with small momentum compaction factor. We could just as easily have chosen other examples.

Low emittance solutions. - Emittance is an important parameter because photon brightness depends inversely upon its value. In Fig. 3 we plot the emittance for each stable lattice setting. The emittances range over 5 orders of magnitude from a few $\mathrm{nm}$ rad to about $0.1 \mathrm{~mm} \mathrm{rad}$. One sees that several regions support low emittance $(<10 \mathrm{~nm} \mathrm{rad}$. The current operating region, region 1 , supports small emittance but so do other regions - regions 2, 3, 4, 5, 7, 8, and 10. Even though all these lattices share the property of small emittance, some of their other properties are very different which may have some potential advantages over region 1 . We will come back to the uniqueness of the different solutions later. We shall now consider another property, namely, the momentum compaction factor.

Small momentum compaction factor solutions. - The momentum compaction factor is an important quantity

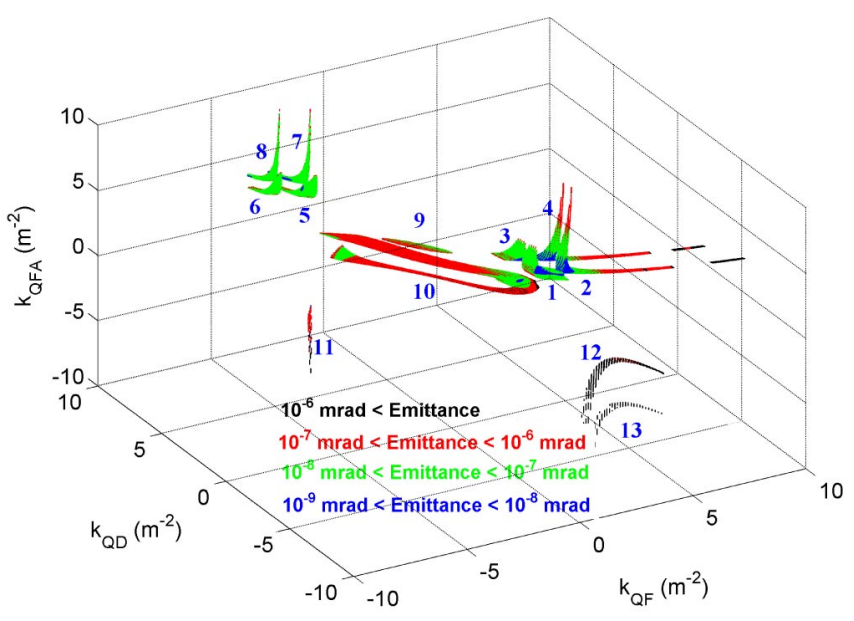

FIG. 3. (Color) Emittance of all linear stable solutions in plotted $k$-space for one of the 12 ALS TBA sectors.

because the bunch length and longitudinal bunch shape depend upon its value. As with the previous example, we plot the momentum compaction of stable solutions in Fig. 4. For an ALS lattice the values of the momentum compaction factor can be positive or negative and range from -0.004 to 0.008 . The nominal ALS lattice momentum compaction is 0.00137 . There are lattices in 3 regions that support small momentum compaction values-regions 2, 4, and 10. In fact, in region 10 there are two small momentum compaction areas. Discovering all these different regions was a surprise. Previously we had known of only one of these regions - one of the two areas in region 10.

Even though each of these lattices shares the property of small momentum compaction, some of their other properties are rather different. For instance, some have small emittance while others have large.

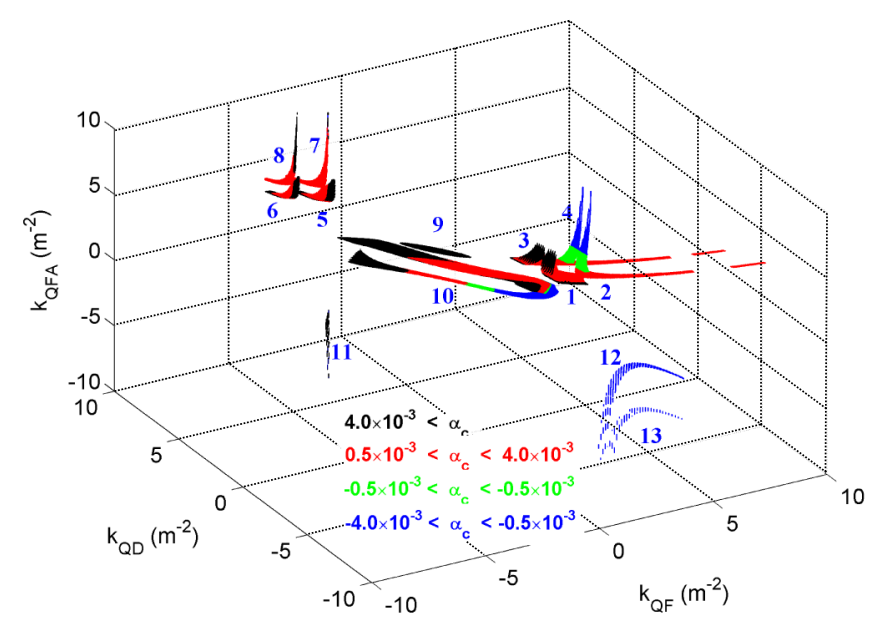

FIG. 4. (Color) Momentum compaction of all linear stable solutions in plotted $k$-space for one of the 12 ALS TBA sectors. 


\section{Improved global understanding}

We have now shown through GLASS how it is very easy to find all possible solutions that have specific properties. Next we will use GLASS to illustrate the characteristics of the different solutions.

Separation by betatron tune. - We can use the computed properties to try to further understand the stability regions. In Fig. 5 the betatron tunes of all stable lattices are plotted. As seen in the figure there are stable solutions covering a large range per sector - from 0.5 to 2 horizontally and 0 to 2 vertically. The nominal tune of the ALS sector is (1.19, $0.77)$.

The tune space can be separated into regions that are $0.5 \times 0.5$ wide. Looking at Fig. 5 , there are 8 half-integersquare regions in tune space where there are solutions. Each of these $0.5 \times 0.5$ regions has been plotted in a different color. The colors will be used to connect the different regions in tune space with the different regions in $k$-space. The color scheme in Fig. 5 is then mapped back into $k$-space in Fig. 6. In Fig. 7 the same $k$-space data is shown from two different perspectives - projecting on $k_{\mathrm{QF}}$ and $k_{\mathrm{QD}}$ plane (left) and projecting on the $k_{\mathrm{QF}}$ and $k_{\mathrm{QFA}}$ plane (right).

It can be seen that the distinct regions in Figs. 5 and 6 can be separated by tune. Sometimes two regions in $k$-space have the same tune. For instance, the 4 tune regions spanning horizontal tunes of 0.5 to 2 and vertical tunes of 0 to 2 (yellow, green, blue, and magenta regions) cover 8 regions in $k$-space. Figure 6 shows a doubling of the number of solutions corresponding to switching the polarity of the QF/QD doublet. The nominal lattice of the ALS has $k_{\mathrm{QF}}$ positive and $k_{\mathrm{QD}}$ negative, which is in the yellow region in tune space and in region 1 in $k$-space.

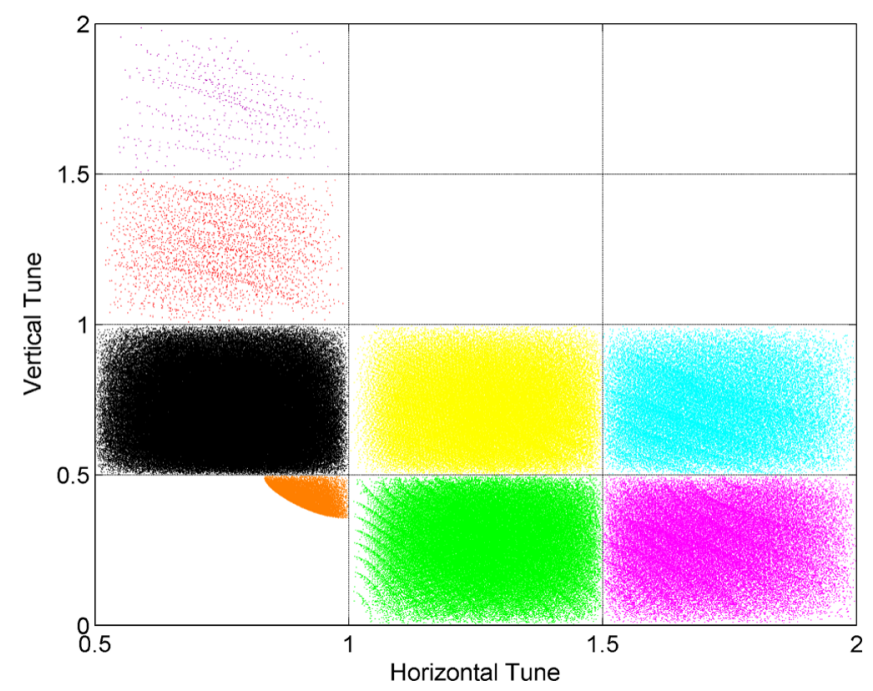

FIG. 5. (Color) All stable solutions plotted in tune space for one of the 12 ALS TBA sectors. Note the nominal ALS sector tune is $(1.19,0.77)$. Each of the half-integer-square regions in tune space is given a distinct color.

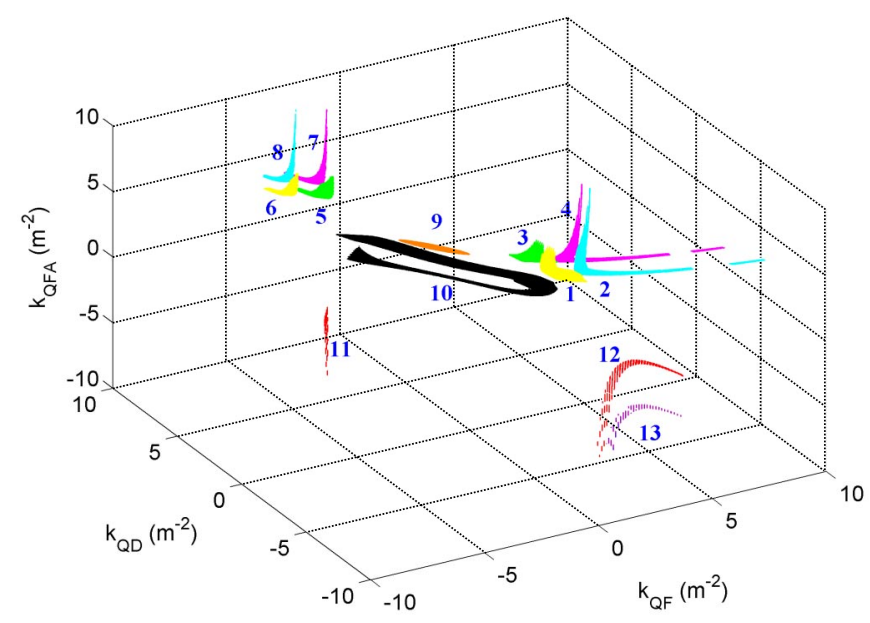

FIG. 6. (Color) The color mapping scheme in Fig. 5 is mapped into $k$-space for one of the 12 ALS TBA sectors.

\section{Properties of some of the ALS regions}

From the earlier discussions, it is known that regions 1 , 2,3 , and 4 can support low emittance. Also, regions 2 and 4 can support small momentum compaction. An examination of the Twiss and dispersion functions leads to a better understanding of these four regions.

Low emittance solutions. - An example of a stable solution in each of these four regions is shown in Fig. 8, and in Table I we provide some data on each. As seen in these figures there are distinct qualitative differences in the Twiss and dispersion functions. The horizontal $\beta$-functions are very similar for regions 1 and 3 with large values in the center of the straight section, whereas in regions 2 and 4 the horizontal $\beta$-functions in the center of the straight are smaller. The lower horizontal $\beta$-function generates the higher horizontal tunes of regions 2 and 4 . On the other hand, the vertical $\beta$-functions are similar in regions 1 and 2 with a small value in the central bend, whereas in regions 3 and 4 the vertical $\beta$-function is large in the central bend. Again this small $\beta$-function in the central bend produces the larger vertical tunes in regions 1 and 2. Each of these solutions is different. This illustrates that, depending on the beam size or divergence required in the straights or arcs, this analysis would identify the optimal regions.

Figure 8 also shows that the dispersion function is different in regions 2 and 4 compared with regions 1 and 3. In 2 and 4 the dispersion function is slightly negative in the outer bends where it is positive in regions 1 and 3 . This negative dispersion function makes it possible to adjust the momentum compaction to small or even negative values in regions 2 and 4 but not in regions 1 and 3 . The possibility of operating the ALS in a low emittance, low momentum compaction optics mode is attractive. In the future we will investigate the feasibility of operation in these regions.

Two very different regions. -An examination of the black region-region 10 - is interesting. One feature of 
(a)

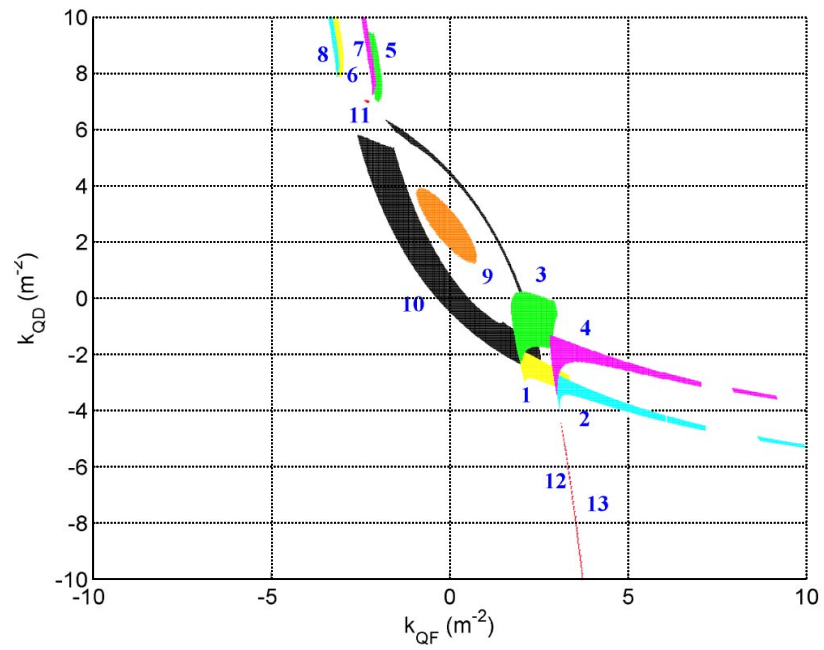

(b)

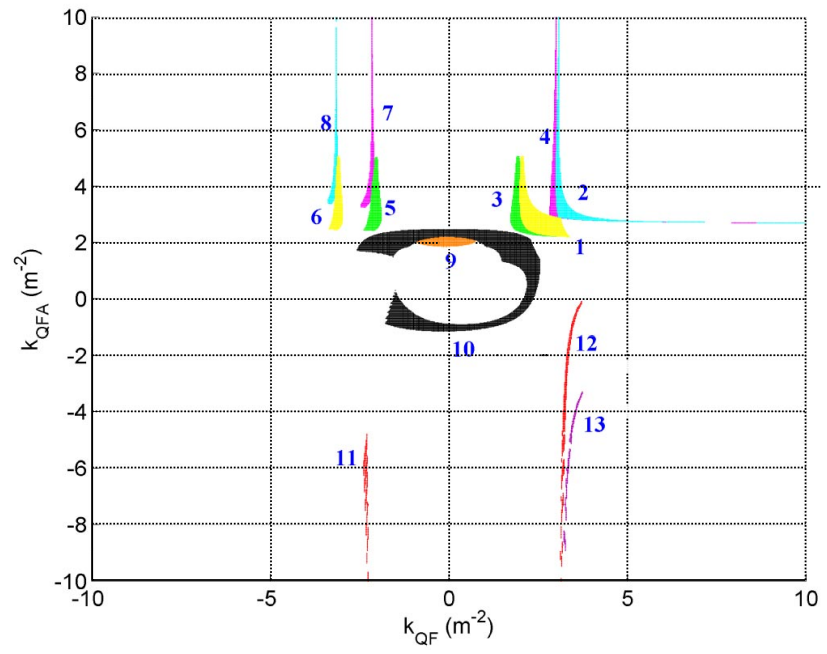

FIG. 7. (Color) $k$-space data shown in Fig. 7 is shown from two different perspectives_-projecting on the kQF and kQD axis (left) and projecting on the $\mathrm{kQF}$ and $\mathrm{kQFA}$ axis (right).
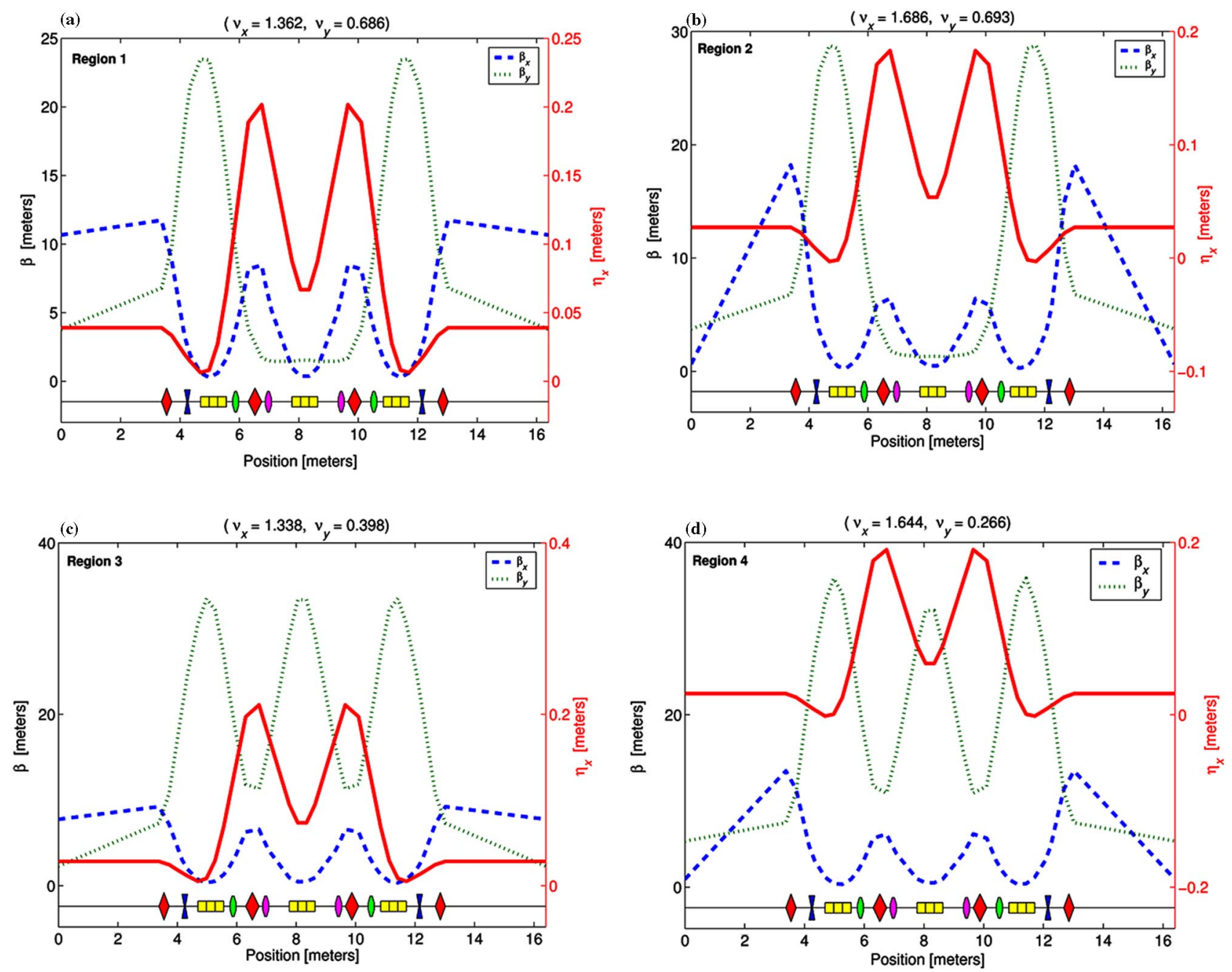

FIG. 8. (Color) A lattice of one of 12 ALS sectors and associated lattice functions in region 1 (top left), region 2 (top right), region 3 (bottom left), and region 4 (bottom right). 
TABLE I. Lattice parameters for displayed ALS lattices.

\begin{tabular}{lcccccc}
\hline \hline Region & $\begin{array}{c}k_{\mathrm{QF}} \\
{\left[\mathrm{m}^{-2}\right]}\end{array}$ & $\begin{array}{c}k_{\mathrm{QD}} \\
{\left[\mathrm{m}^{-2}\right]}\end{array}$ & $\begin{array}{c}k_{\mathrm{QFA}} \\
{\left[\mathrm{m}^{-2}\right]}\end{array}$ & $\nu_{x}$ & $\nu_{y}$ & {$\left[\mathrm{~m} \mathrm{rad} \times 10^{-9}\right]$} \\
\hline 1 & 2.48 & -2.56 & 3.02 & 1.362 & 0.686 & 4 \\
2 & 3.2 & -3.08 & 3.18 & 1.686 & 0.693 & 3 \\
3 & 2.32 & -1.44 & 2.98 & 1.338 & 0.398 & 5 \\
4 & 3 & -1.84 & 3.12 & 1.644 & 0.266 & 3 \\
9 & -0.40 & 3.12 & 2.08 & 0.954 & 0.399 & 99 \\
10 & 2.08 & -1.60 & 1.18 & 0.594 & 0.737 & 190 \\
\hline \hline
\end{tabular}

some lattices in this region is that it is also possible to operate with a low or even negative momentum compaction. This was previously known [8]. As one can see from the sector plotted in Fig. 9, it is very different from the low momentum compaction lattices in regions 2 and 4 (see Fig. 8). In region 10 the dispersion is positive in the outer bends and negative in the inner one.

The GLASS analysis also revealed some very curious effects about region 10. One such curiosity is that in the black region it is possible to turn off either the QFA or both the QF and QD magnets and still be stable! This can be seen in Fig. 7. This fact is probably of little practical interest but is nevertheless a interesting and counterintuitive result.

The final lattice that we show for the ALS is a lattice in region 9 whose functions are shown in Fig. 10. This is also a stable region that was previously unknown. It has very small tunes in each plane and the lattice functions are rather different from other regions that have been presented. The $\beta$-functions and dispersion look very different from those presented before. It is a region with reasonably large emittance (see Fig. 3) but might be of interest for some applications. The purpose of showing this region is to illustrate that the lattice has a lot of flexibility and diversity

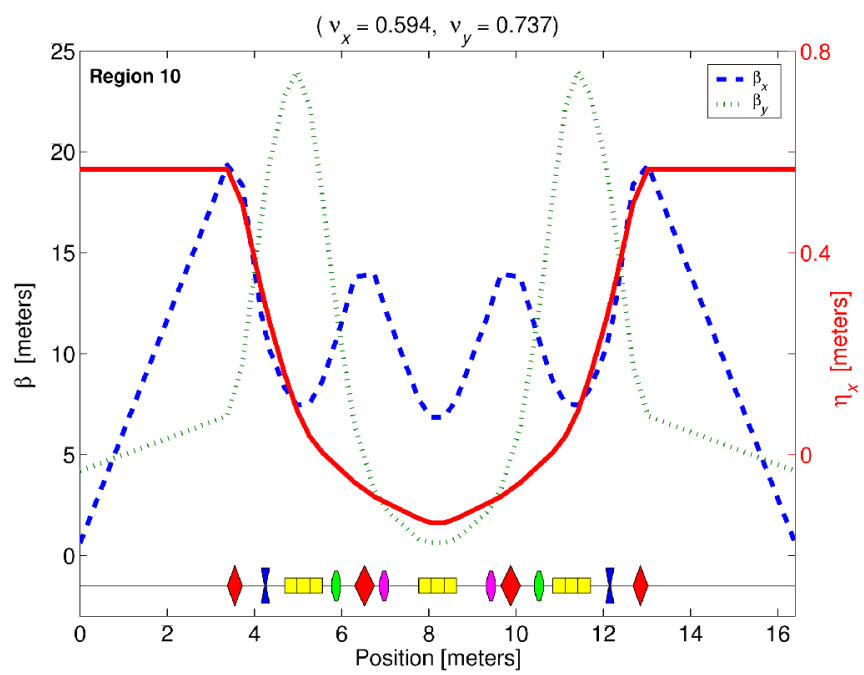

FIG. 9. (Color) Lattice of one of 12 ALS TBA sectors and associated lattice functions in region 10 .

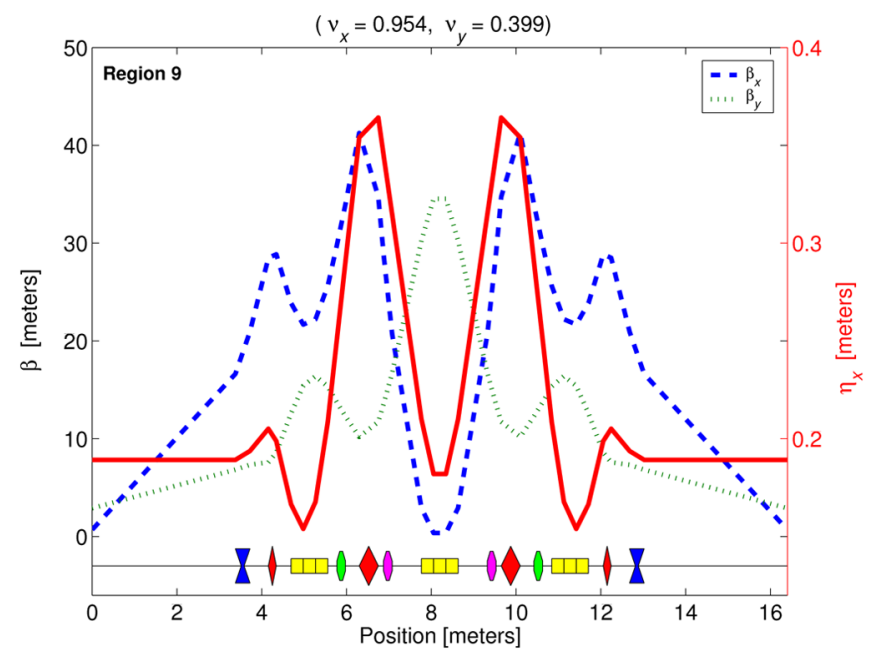

FIG. 10. (Color) Lattice of one of 12 ALS TBA sectors and associated lattice functions in region 9.

in the settings that it can support. One can continue the analysis for the other regions.

\section{B. Example 2-double bend achromat of CAMD}

Now we will briefly show some results from a different lattice structure - the double bend achromat structure of the CAMD Light Source at Louisiana State University.

Similar to the TBA, the DBA is another common lattice structure that is used in many machines. Here we take one example - that of CAMD - and apply the GLASS technique to it.

The CAMD lattice consists of 4 double bend achromat sectors. In Fig. 11 we show the structure and Twiss parameters [9] for one of those sectors. As seen in Fig. 11, the

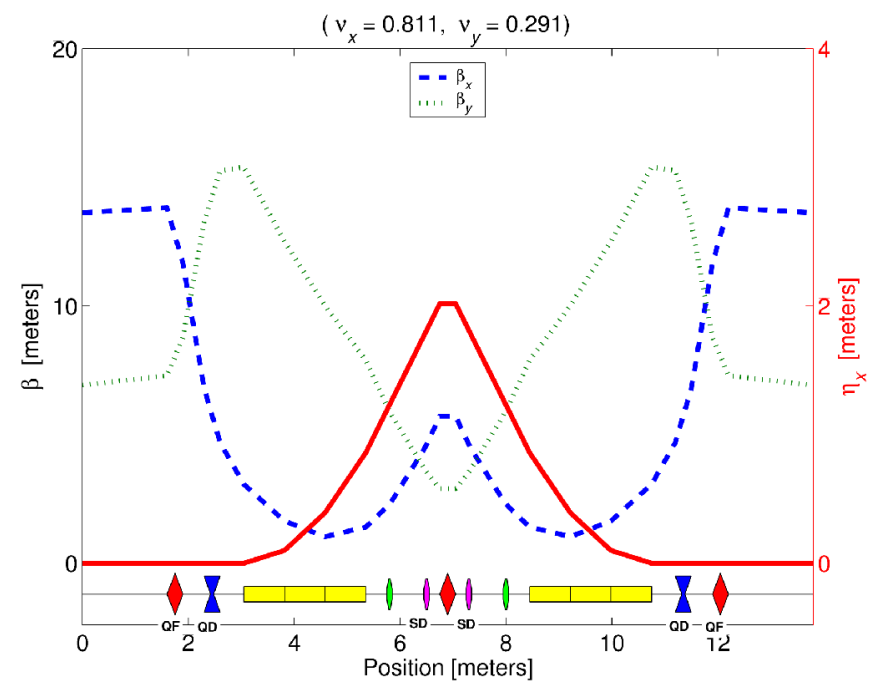

FIG. 11. (Color) Lattice of one of 4 CAMD sectors and associated lattice functions in a nominal operational mode starting from the center of one straight section and going to the center of the following straight section. 


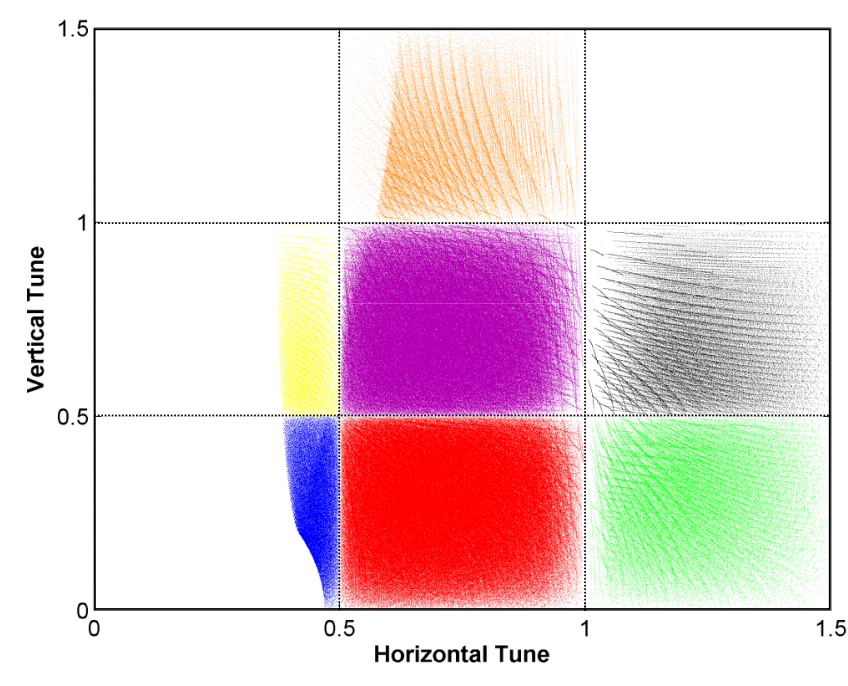

FIG. 12. (Color) Using different colors to illustrate how the different regions in tune space for one of the four CAMD DBA sectors correspond with the different regions in $k$-space. Each of the $0.5 \times 0.5$ regions in tune space is given a distinct color.

structure consists of 2 dipoles, and three quadrupoles - QF, QD, and QA. For these settings shown in Fig. 11, the CAMD sector operates as an achromat, with tunes $\left(\nu_{x}, \nu_{y}\right)=(0.811,0.291)$ and dispersion at the center of the long straight sections, $\eta=0.0[\mathrm{~m}]$.

For all 3 quadrupole families, the magnet strengths $k_{\mathrm{QF}}$, $k_{\mathrm{QD}}$, and $k_{\mathrm{QA}}$ are each scanned over 500 settings ranging between $k=-5 \mathrm{~m}^{-2}$ and $5 \mathrm{~m}^{-2}$. As in the previous example, we found that it was instructive to look at the results in tune space. The results are plotted in Fig. 12 where the different half integer regions are plotted in color (similar to Fig. 5 for the ALS TBA sector). As in Fig. 5 we see that there are several half-integer-square regions that contain

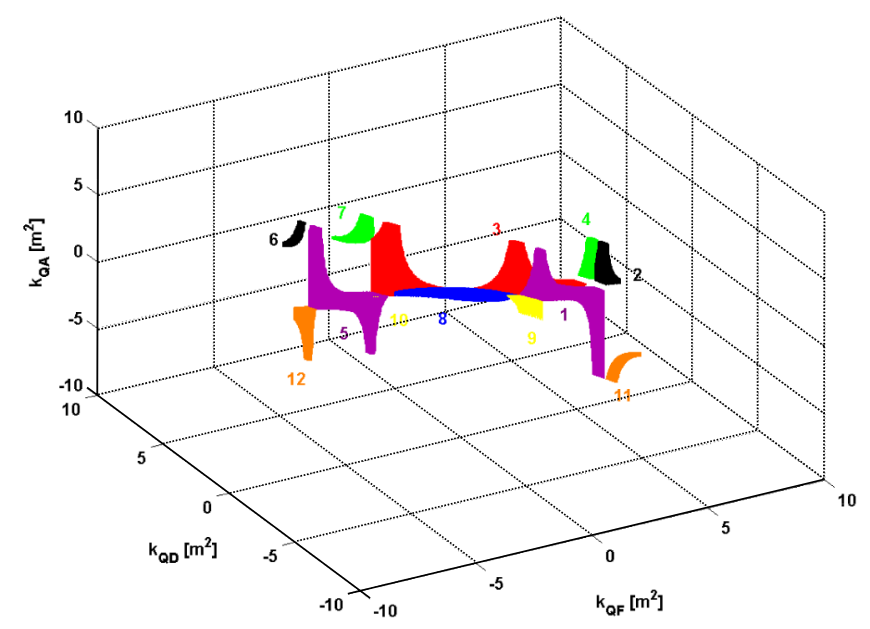

FIG. 13. (Color) The color mapping scheme in Fig. 12 is mapped into $k$-space. stable solutions. They are somewhat different from those for the ALS TBA.

In a similar manner to what was done for the TBA lattice (see Fig. 6), in Fig. 13 we plot the stable solutions in $k$-space in color. The color mapping is determined by the tune space as plotted in Fig. 12. There are approximately 12 distinct regions of stability in $k$-space but the shape and locations of the regions are much different than for the ALS TBA lattice. In Fig. 14 the same $k$-space data is shown from two different perspectives - projecting on the $k_{\mathrm{QF}}$ and $k_{\mathrm{QD}}$ plane (left) and projecting on the $k_{\mathrm{QF}}$ and $k_{\mathrm{QA}}$ plane (right).

\section{Low emittance solutions}

Consider the low emittance regions for CAMD operating at the energy of $1.3 \mathrm{GeV}$. In Fig. 15 we plot the emittance for each stable setting. The emittance ranges over 3 orders of magnitude from about $46 \mathrm{~nm}$ rad to about $0.1 \mathrm{~mm} \mathrm{rad}$. One sees that several regions support low emittance $(<100 \mathrm{~nm} \mathrm{rad})$ lattices. The current operating region 3, supports small emittance, but so do other regions-regions $1,2,3,4$, and 7. As was seen for the case of the ALS TBA (Fig. 8), there are large differences in the shape of the lattice functions for the different regions.

In particular, consider solutions that are both low emittance and achromatic $(\eta=0 \mathrm{~m}$ in the long straight section). In Fig. 16 we plot one lattice in each of the four low emittance regions $(1,2,3,4)$ for CAMD. In Table II we provide some data on each lattice. As seen in Fig. 16 and Table II, there are distinct qualitative differences in the Twiss and dispersion functions. The horizontal $\beta$-functions are very similar for regions 1 and 3 with large horizontal $\beta$-functions in the center of the straight section, whereas in regions 2 and 4 the horizontal $\beta$-functions in the center of the straight are smaller. The lower horizontal $\beta$-function produces higher horizontal tunes in regions 2 and 4 . On the other hand, the vertical $\beta$-functions are similar in regions 1 and 2 with a small vertical $\beta$-function in the straight section (and also in the central quadrupole), whereas in regions 3 and 4 the vertical $\beta$-function is larger in the straight sections (as well as in the central quadrupole). Again this small $\beta$-function in the straight section results in larger vertical tunes in regions 1 and 2. This illustrates that, depending on what beam size or divergence one would like in the straights or arcs, this analysis would help to guide one to the optimal regions. In fact CAMD is preparing to move the nominal operating region from one in region 3 to one in region 1 . The reason for the change is to operate with a reduced vertical $\beta$-function in the straight section to minimize the impact of a proposed high field multipole wiggler [10]. It should be noted that this new working point was discovered by the GLASS technique. 
(a)

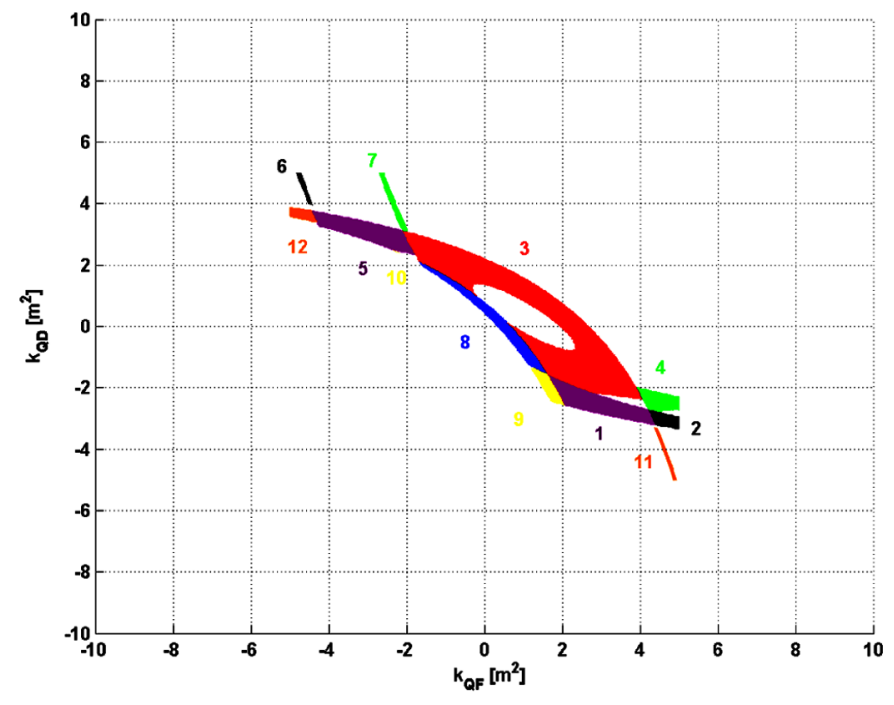

(b)

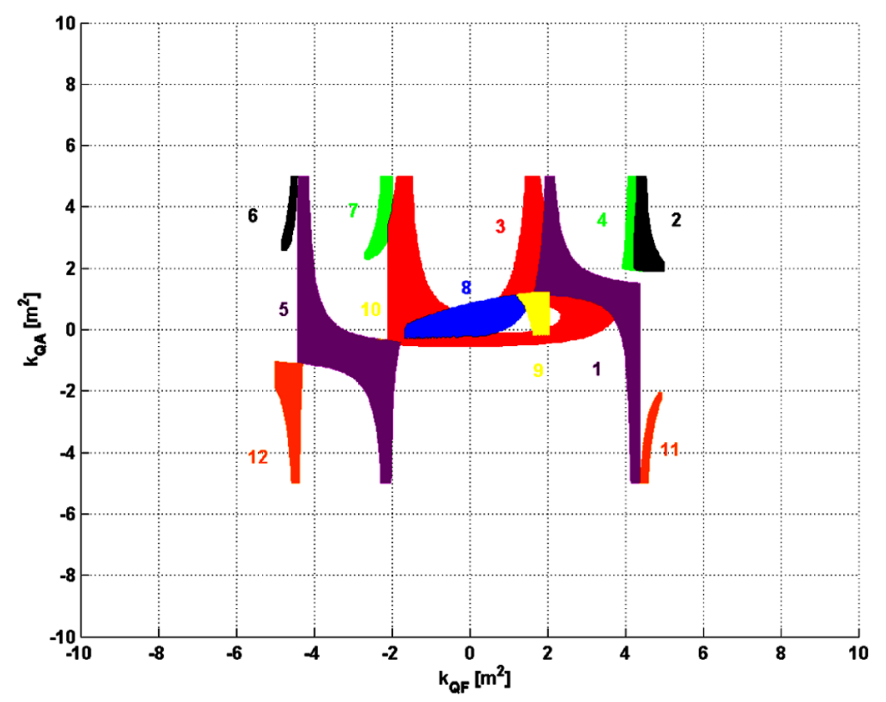

FIG. 14. (Color) $k$-space data shown in Fig. 13 is shown from two different perspectives - projecting on the kQF and kQD axis (left) and projecting on the $\mathrm{kQF}$ and $\mathrm{kFA}$ axis (right).

Finally, we present a solution from region 7 in Fig. 17 and in Table II. In this region the polarity of QF and QD quadrupoles is reversed. We see that in this region we have a very small vertical $\beta$-function in the straight and a larger horizontal $\beta$-function in the straight. The actual values of the $\beta$-functions get somewhat large and probably make it impractical for operation but the shape is interesting and has many of the qualitative features that one might like for a low emittance lattice - this analysis for low emittance lattices is just an illustration of how one could use GLASS.

\section{EXTENSION OF GLASS}

The GLASS analysis is general and can be extended to more than 3 parameters. This is desirable because many

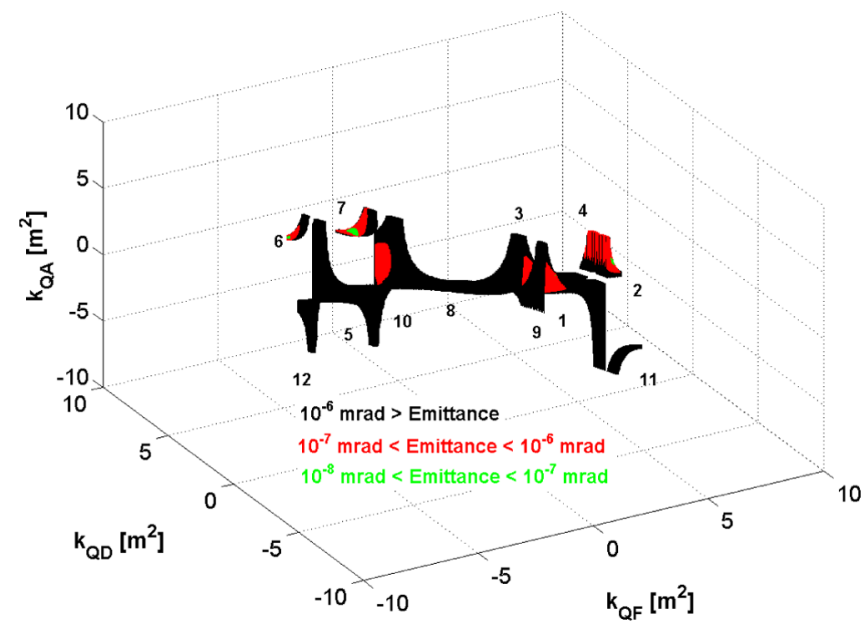

FIG. 15. (Color) Emittance of all linear stable solutions of one of the four CAMD DBA sectors plotted in $k$-space. storage ring lattices consist of more than 3 quadrupole families. One could also consider extending the technique for the investigation of nonlinear effects such as sextupole strengths, amplitude dependent tune-shifts, and so on. In addition, it is possible to improve upon the data mining process to obtain a better understanding of the optimal tradeoffs. Below we discuss ways of how the GLASS process can be extended.

\section{A. Increasing the number of parameters}

An obvious concern when increasing the number of scanned parameters is the increase in computing time. There are several ways to deal with that. The brute force was is to use more computing power or time. Also one could easily imagine a somewhat simple minded approach where several iterations are performed - the earlier iterations using a large grid spacing to locate the stability regions and then subsequent iterations to make finer step sizes to more carefully map out the stability regions. This is a simple extension.

In addition there are more sophisticated global search methods, such as the so-called multiobjective evolutionary algorithms, that are well suited to this type of problem. Below we will briefly discuss what they are and how they may be useful.

\section{Multiobjective evolutionary algorithms}

Multiobjective evolutionary algorithms, (MOEA) are relatively new optimization algorithms, which have achieved notable successes in finding global optimums and tradeoffs for problems in science, commerce, and engineering. MOEA do not use any gradient information (no knowledge of the derivative of the objectives with 

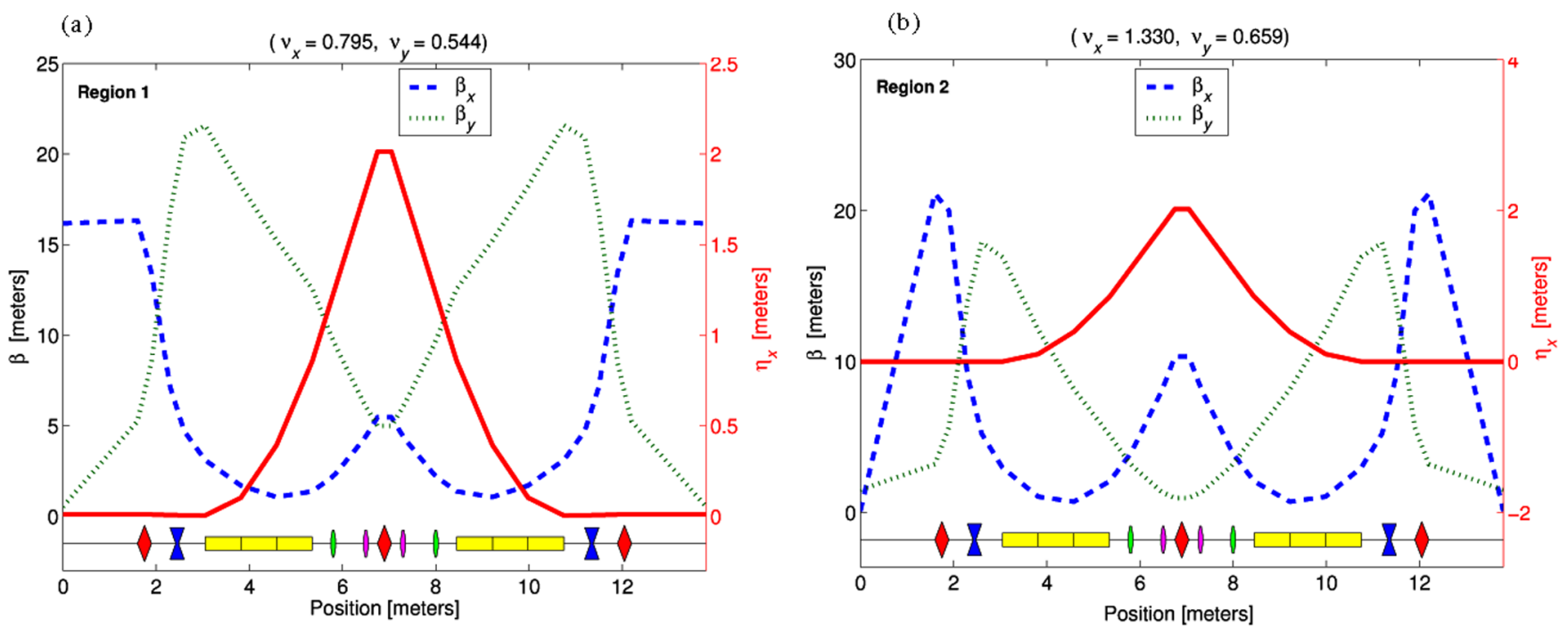

(c)

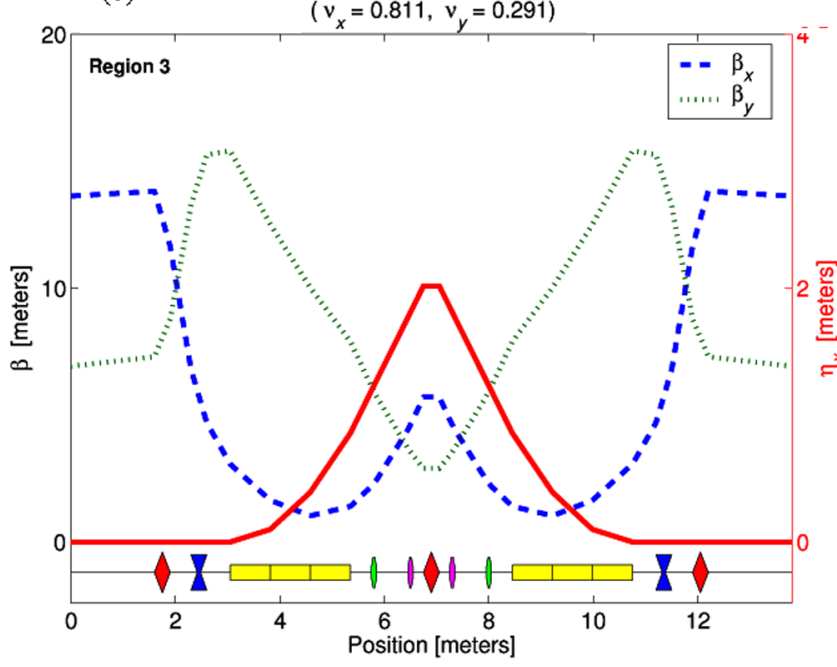

(d)

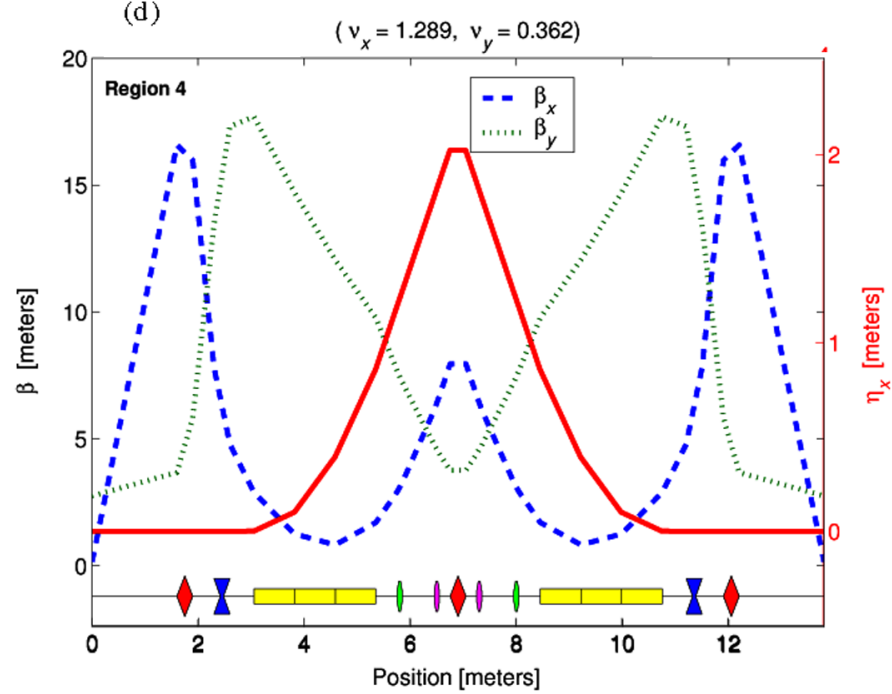

FIG. 16. (Color) A lattice of one of four CAMD DBA sectors and associated lattice functions in region 1 (top left), region 2 (top right), region 3 (bottom left), and region 4 (bottom right).

respect to the parameters is required) and in many cases mimic the natural selection process. They are robust, efficient, and widely applicable in optimization problems and work well for discretely continuous solutions (unconnected sets). This is just the type of problem one faces in lattice optimization where there are islands of stability. MOEA

TABLE II. Lattice parameters for displayed CAMD lattices.

\begin{tabular}{|c|c|c|c|c|c|c|}
\hline Region & $\begin{array}{c}k_{\mathrm{QF}} \\
{\left[\mathrm{m}^{-2}\right]}\end{array}$ & $\begin{array}{c}k_{\mathrm{QD}} \\
{\left[\mathrm{m}^{-2}\right]}\end{array}$ & $\begin{array}{c}k_{\mathrm{QA}} \\
{\left[\mathrm{m}^{-2}\right]}\end{array}$ & $\nu_{x}$ & $\nu_{y}$ & $\begin{array}{c}\varepsilon_{x} \\
{\left[\mathrm{~m} \mathrm{rad} \times 10^{-9}\right]}\end{array}$ \\
\hline 1 & 2.14 & -2.12 & 2.68 & 0.795 & 0.544 & 300 \\
\hline 2 & 4.62 & -3.02 & 2.68 & 1.330 & 0.659 & 190 \\
\hline 3 & 1.90 & -1.46 & 2.68 & 0.814 & 0.294 & 290 \\
\hline 4 & 4.42 & -2.54 & 2.68 & 1.289 & 0.362 & 225 \\
\hline 7 & -4.78 & 4.94 & 2.68 & 1.133 & 0.822 & 195 \\
\hline
\end{tabular}

work with a population of solutions at each iteration and utilize stochastic and deterministic operators for the evolution of the population. A successful application of such algorithms in accelerator physics was toward the optimization of a high brightness dc gun photoinjector [11]. As a result, MOEA are well suited to lattice optimization. Below we present an overview of the most salient features of these algorithms. For a more detailed description the reader is referred to [12-14].

The general problem one wants to solve with MOEA is to optimize one or more sets of objectives while satisfying some constraints. In our case, the constraint could be linear stability and the objectives the optimization of quantities such as emittance, momentum compaction, $\beta$-functions, and so on. There are many different evolutionary algorithms but they typically go through the same process. One begins with a large number of initial trial solutions. The 


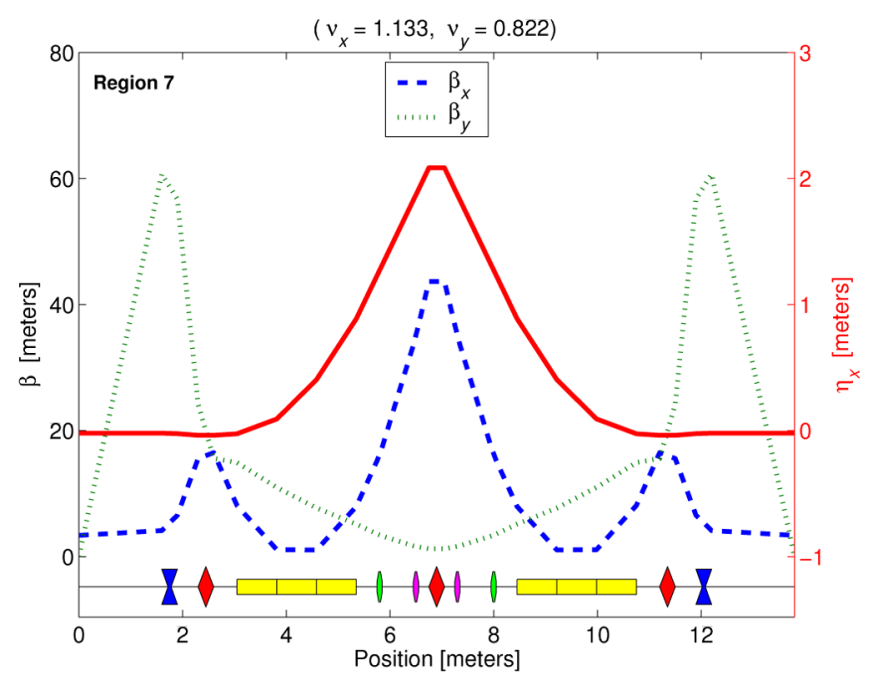

FIG. 17. (Color) Lattice functions for a CAMD DBA sector in region 7. The polarities of the QF and QD magnets are reversed. This example was chosen to illustrate an achromat with relatively small emittance.

following steps are then performed for each iteration to generate a new population: (1) selection, (2) crossing, and (3) mutation. In the selection process the constraints and objective functions are evaluated for the population. A fitness value is assigned to each member of the population based on a comparison relation. A selection operator is then applied to create a mating pool of some fixed size with a preference for solutions with better fitness. A deterministic crossing operator then generates new offspring solutions, and a stochastic mutating operator can be used to diversify the population. If the process is done correctly, the population of solutions moves towards the so-called Pareto-Optimal set that contains all the "best" solutions, trading between the different objectives of the optimization problem. MOEA have proven to be very efficient from the computing time point of view, allowing to optimize many parameter-family problems where the use of a brute force systematic-scan approach can be completely unthinkable [11].

In our case, one could imagine using MOEA in the first step of the GLASS process as a more efficient way of locating and mapping the stable regions than a simple but large grid search. But once the stable regions are found, then one could populate them with points to create a database of solutions readily accessible in a later time. Or if one is primarily interested in understanding the tradeoffs of some specific objectives (say emittance versus momentum compaction), then one could apply multivariant evolutionary analysis to aid in the data mining process. From the technical point of view, the success of GLASS is based largely on the fast evaluation of the transfer matrices. It is conceivable that we use the same tool for MOEA to increase the speed of the optimization process. In the end,
MOEA and GLASS may be organically combined to form a new tool of optimization.

\section{CONCLUSION}

A newly extended technique GLASS allows one to see all possible linear stable solutions and associated properties for a given simple lattice. It has been shown that, by using GLASS, one can uncover many interesting and previously unknown stability regions. In a sense, GLASS functions as a lattice observatory clearly displaying all possibilities. Already GLASS has located a superior operational point for CAMD that will be used for operation in the near future. GLASS is an example of a global analysis technique which gives the lattice designer clear guidance as to where to look for interesting operational points. The technique is robust and global. The examples given were for lattices with 3 parameters. Extension to more complex lattices and improved data mining should be possible using more sophisticated evolutionary algorithms.

\section{ACKNOWLEDGMENTS}

This work was supported in part by the Director, Office of Energy Research, Office of Basic Energy Sciences, Material Sciences Division, U.S. Department of Energy, under Contract No. DE-AC03-76SF00098. The authors are grateful to Michael Borland and Louis Emery for introducing them to the field of multivariant evolutionary algorithms.

[1] E. D. Courant and H. S. Snyder, Ann. Phys. (Leipzig) 3, 1 (1958).

[2] J.A. Clark et al., 1992 European Particle Accelerator Conference, p. 679.

[3] V. Suller et al., 2004 European Particle Accelerator Conference, Lucerne, Switzerland, p. 2421.

[4] H. Nishimura et al., 2007 Particle Accelerator Conference, Albuquerque, NM, p. 1170.

[5] E. D. Courant, M. S. Livingston, and H. Snyder, Phys. Rev. 88, 1190 (1952).

[6] M. Berz, J. Hoefkens, and K. Makino, Report No. MSUHEP-20703, Department of Physics and Astronomy, Michigan State University, 2002.

[7] See, for instance, H. Wiedemann, Particle Accelerator Physics (Springer-Verlag, Heidelberg, 2003), Vol. I.

[8] D. S. Robin, R. Alvis, A. Jackson, R. Holzapple, and B. Podobedov, Proceedings of the Microbunches Workshop, Upton, NY, 1995, AIP Conf. Proc. No. 367 (AIP, New York, 1996), p. 181.

[9] J. Murphy, Report No. BNL-42333-(Rev.05/96), Brookhaven National Laboratory, pp. 100-101.

[10] V. P. Suller, M. Fedurin, P. Jines, D. Launey, T. Miller, and Y. Wang, 2007 Particle Accelerator Conference, Alburquerque, NM, pp. 1161-1163.

[11] I. Bazarov and C. Sinclair, Phys. Rev. ST Accel. Beams 8, 034202 (2005). 
[12] T. Bäck, Handbook of Evolutionary Computation (Oxford University Press, Oxford, 1997).

[13] K. Deb, Multi-Objective Optimization using Evolutionary Algorithms (Wiley, Chichester, 2001).
[14] C. A. C. Coello, D. A. V. Veldhuizen, and G. B. Lamont, Evolutionary Algorithms for Solving Multi-Objective Problems (Kluwer Academic/Plenum, New York, 2002). 